\section{Bernt Schnettler}

Bayreuth University, Germany

\section{Bernd Rebstein}

Bayreuth University, Germany

\section{International Perspectives on the Future of Qualitative Research in Europe}

DOI: https://doi.org/10.18778/1733-8077.8.2.01
$\mathrm{O}$ r the past decades, Qualitative Reearch has repositioned itself within the discipline of sociology. Today, a large variety of Qualitative Methods is used in a broad range of research areas. There is no doubt that they have succeeded in overcoming their marginalization, and it can even be claimed that Qualitative Research has attained a somehow privileged position. Presently, we can witness a growing number of scientific contributions to a large variety of fields and specialties based in Qualitative Research; there are numerous sessions, workshops and conferences each year, not only in Europe but globally, and scientific associations and research networks dedicated to Qualitative Research are quickly growing. Moreover, the emergence of several high-impacts journals and the institutional expansion of chairs, readers, and other permanent university positions with this specification evidently reflect that Qualitative Research has, indeed, become established.

However, its growth and success deliver not only new opportunities, but also new challenges. As is well known, the impact of Qualitative Methods varies from discipline to discipline and we still observe manifest local and national differences. Achievements are not evenly distributed and we are faced with an excitingly complex and varied landscape if one tries to figure out the current state of Qualitative Research in Europe. To discuss the current state of Qualitative Research in Europe and its perspective for the immediate future was precisely the purpose of the midterm conference of the European Sociological Association (ESA) Research Network Qualitative Methods which took place in Bayreuth,
Germany, in September 2010. Consequently, the conference focused on vital problems related to the future of Qualitative Methods in European sociology, as well as in neighboring disciplines. The papers presented and the discussions dealt with questions concerning methodological in novations and the contributions of Qualitative Methods to substantive areas of research, as well as to sociological theory. The overall goal was to develop further and promote Qualitative Methods within an emerging European research realm while fostering exchange with researchers and scholarly networks in other world regions.

Organized by the Chair of Sociology of Culture and Religion of the University of Bayreuth (Bernt Schnettler), the event had a remarkably international attendance. For two days, intense discussions were held between more than 90 scholars and researchers from Germany and many other European countries, as well as from the USA, Latin America and Africa. Within this framework, numerous contributions to Qualitative Methods were presented from within a broad spectrum of research areas, including religion, new technologies, migration, ethnography, research on Africa, grounded theory, discourse analysis, urban sociology and community studies, as well as social memory studies. ${ }^{1}$

In this special issue, we publish selected papers based on presentations given at the conference.

${ }^{1}$ A detailed report about the sessions and the whole conference activities, including a short video documentation, can be accessed at: http://www.soz.uni-bayreuth.de/ de/conferences/ESAmidterm2010/Report/index.html. 
In addition, we include a documentation of the inaugural addresses delivered by Krzysztof Konecki and Bernt Schnettler, along with the comprehensive transcription of the two plenary sessions on "The Future of Qualitative Research in Europe" dedicated to discussions concerning the conference's leading question.

It was in these two plenary sessions where a number of renowned international scholars from several European countries - each of them an outstanding specialist in Qualitative Methods - discussed with colleagues from the U.S., Africa and Latin America about the forthcoming challenges and risks for Qualitative Research in Europe. ${ }^{2}$ Qualitative Research in Europe operates in an increasingly interconnected research space. The density of cooperation between researchers from various countries and across disciplines has significantly developed over the past years. Participants in the plenary session discussed how Qualitative Methods can be strengthened in Europe without stepping into the trap of standardization and mainstreaming. They also debated about the social relevance of this kind of research and how it may contribute to solutions for social problems. And they asked in what way we can work against the marginalization of non-mainstream Qualitative

${ }^{2}$ The first plenary was moderated by ESA Research Network Qualitative Methods ( $R N$ 20) president Krzysztof Konecki and included César Cisneros (Mexico City), Thomas Eberle (St. Gall), Hubert Knoblauch (Berlin), Elisio Macamo (Basle), David Silverman (London), and Miguel Valles (Madrid) as speakers. The second, chaired by Bernt Schnettler, comprised scholars including Jan Coetzee (Rhodes University, South Africa), Giampietro Gobo (Milan, Italy), Krzysztof Konecki (Lodz, Poland), Anne Ryen (Agder, Norway), Jörg Strübing (Tubingen, Germany) an Ruth Wodak (Lancaster, UK and Vienna, Austria).
Traditions - e.g., from Spain and Latin America - in an Anglo-centric academic world. The speakers held diverse opinions about the role of theoretical fundaments and the relevance of an appropriate methodological basis for Qualitative Research. However, they unanimously and strongly supported the claim for autonomy of Qualitative Methods and the need to enhance our efforts of making the results of Qualitative Research better known to the wider society, as well as to our colleagues in sociological theory. The discussants also encouraged the RN to increase its intents of including scholars from Eastern European countries who are still underrepresented. They also commented on the possibility of involving sociology's neighbor disciplines in order not only to strengthen interdisciplinary research, but also to develop further methodological innovations and the interdisciplinarity of Qualitative Methods.

The fact that the conference had to cope with the absence of two plenary speakers opened the space for an experiment: Katja Mruck (Berlin) and Günter Mey (Stendal) delivered their statements via video message, introducing their online journal project called Forum Qualitative Sozialforschung / Forum: Qualitative Social Research (FQS). This experiment vividly demonstrated the new opportunities of mediated forms of communication and multi-site-collaboration, the advantages of computer supported distributed research and the leading role that audio-visual analysis plays in this respect. However, the limitations of this mediated participation was wellexpressed by the lack of sharing the co-presence live experiences typical for meetings and con- ferences with our two absent colleagues. This is only partly cured by the fortunate fact that one can repeatedly access Mruck's and Mey's video presentation online (for the link, see p. 202 [Documentation of the 2010 Midterm Conference of the European Sociological Association Research Network 20 Qualitative Methods "Innovating Qualitative Research: Challenges and Opportunities. New Directions in Religion, Technology, Migration and Beyond" - in this issue of Qualitative Sociology Review]).

\section{Contributions to this Special Issue}

The papers, debates and discussions at the midterm conference were framed by the challenges and opportunities for Qualitative Research, finding itself at the crossroad of an on-going development in which it has been established successfully and is now facing an evolving institutionalization. One must assume that in the future Qualitative Methods will have to struggle to preserve their methodological openness and flexibility. In this sense, innovation is, and surely will be, an integral part of the majority of all Qualitative Methods. The recently flourishing book market on Qualitative Research vividly demonstrates the unstoppable appearance of new approaches, procedures and techniques that broaden the scope day-by-day. At this critical moment of becoming part of a disciplinary mainstream, innovation is a special imperative. Innovation is necessarily tied to critical reassessment and reflection of the current state and should include seminal examples that take up the future challenges. Accordingly, the task of the two plenary sessions was to evaluate the current state in order to open the horizons. There are few documents which strive to collect the plurality of perspectives, experiences and voices on Qualitative Research in its richness as carried out throughout Europe and on other continents. Trying to summarize the faceted discussion would be futile and reductive. Instead, we decided to include the entire documentation here (see p. 164).

Another important, critical reflection was done by David Silverman during the conference. In his programmatic keynote-speech, Silverman argued strongly against the shortcomings of an atrophy version of Qualitative Research in which verbal statements, collected in interviews, are taken as "mirror" to the subject's motivations. This frequent error is partly an outcome of a poor understanding and deficient methodological training, which underestimates the role of observable behavior and "naturally" given data. Thus, Silverman advocated improved methods of data-collection and analysis in order to achieve better results. A book based on this is forthcoming (Silverman forthcoming).

Seminal examples of innovative approaches in Qualitative Research are included in this special issue. They have been selected according to their potential for opening up new perspectives. Certainly, this issue can not cover all areas in which stimulating new developments in Qualitative Research are going on. Furthermore, the compilation is probably biased by the editor's subjective preferences; all possible flaws should be attributed to that fact. The selection has also three marked emphases: on ethnography, on 
discourse, and on visual and audio-visual analysis, thereby highlighting some of the outstanding current trends in the field.

Ethnography can still be considered the baseline and "mother" of all Qualitative Methods because of its special dedication to fieldwork and natural data. The article by David Wästerfors, who studies social ties in a residential care institution for juvenile delinquents, Regine Herbrik's work on emotional styles in several Christian churches and Bernd Rebsteins's analysis of communication on social events in the migration milieu, are grounded in extensive ethnographic fieldwork. Also, the analysis of Katharina Inhetveen, who writes on the problems of translation and their methodological implications for conducting Qualitative Research in multi-lingual settings, would have been impossible without her fieldwork in African refugee camps. The same holds for René Tuma's methodological paper on the improvements for interpretive social research from particular everyday life and professional practices, which rely on data taken from "naturally occurring data sessions."

The analysis of discourse - in all its varieties and applications - is, without doubt, one of the leading trends in interpretive social research, with a decisive impact in sociology, history, linguistics and a number of cognate disciplines. Reiner Keller argues for a new programmatic approach that combines Berger's and Luckmann's sociology of social construction with the perspective of Foucault, proposing what he labels as "sociology of knowledge approach to discourse" (SKAD). His inclusive proposal is deeply committed to
Qualitative and Interpretive research traditions in sociology and it shows special potential for bridging the still existing gap between Germanic, Francophone and Anglo-Saxon approaches. Two articles are located at the intersection of discourse and visual analysis. Jan Krasni, dealing with data extracts from media coverage on bonus payments to top bank managers, combines two methods of media discourse analysis in order to achieve new insights into power relations inherent in texts and into the manner in which collective memory is constituted. In her article, Antonia Schmid also takes up the challenge of analyzing visual data, given the fact that pictures and images play a central role in contemporary society by mediating meaning in a seemingly universal environment.

Finally, audio-visual data analysis lies at the core of Herbrig's contribution, who demonstrates how the emotionalization of religion depends on the practices of visualization as presented in the media and on site in several congregations and churches. Her results are part of a multi-method research design, including video-analysis, interviews and participant observation. Rebstein emphasizes the role of contextual information collected in focused ethnographic fieldwork for the interpretation and analysis of videographic data sequences, demonstrating how fieldwork and data analysis go hand-in-hand and methodically depend on each other. Finally, Tuma is specially focusing on the further development of video analysis. He concentrates on a widely neglected topic, namely the "natural practice" of analyzing video data, in order to gain insight into the ways in which non-scientific members deal with video. His article is an impressive exercise in reflexive methodology. By revising the sense-making practices in several contexts of "video in use," he demonstrates how to extract methodological insight from a close look into these practices.

Given its rapid development, richness and diversity, summing up the current state and future prospects for Qualitative Research has become an impossible task. Our purpose is far from drawing comprehensive pictures. The snapshots presented in this issue, however, may serve to engender new debates that continue the on-going duty of innovating and improving our research approaches in Qualitative Methods.

\section{Acknowledgments}

The conference and its documentation in this special issue were made possible by a broad cooperation between various scientific associations. We appreciate the support and funding by the European Sociological Association, the Research Network Migration and Knowledge (ForMig), the University of Bayreuth and the University Association, the Bavarian Ministry for Education, Science and Culture (BMWK), as well as the Ger-

\section{References}

Silverman, David. forthcoming. A Very Short, Fairly Interesting, Reasonably Cheap Book about Qualitative Research. $2^{\text {nd }}$ ed. London: Sage.

Schnettler, Bernt and Bernd Rebstein. 2012. "International Perspectives on the Future of Qualitative Re" search in Europe." Qualitative Sociology Review 8(2):6-11. Retrieved Month, Year (http://www.qualitativesociologyreview.org/ENG/archive_eng.php). 